\title{
Approximating Common Fixed Points for a Finite Family of Asymptotically Nonexpansive Mappings Using Iteration Process with Errors Terms
}

\author{
Seyit Temir ${ }^{1,2}$ and Adem Kiliçman ${ }^{3}$ \\ ${ }^{1}$ Department of Mathematics, Arts and Science Faculty, Harran University, 63200 Şanliurfa, Turkey \\ ${ }^{2}$ Graduate School of Natural and Applied Sciences, Adıyaman University, 02040 Adryaman, Turkey \\ ${ }^{3}$ Department of Mathematics and Institute for Mathematical Research, University Putra Malaysia, 43400 Serdang, Selangor, Malaysia
}

Correspondence should be addressed to Adem Kiliçman; akilic@upm.edu.my

Received 6 October 2013; Accepted 25 November 2013

Academic Editor: Mohammad Mursaleen

Copyright (C) 2013 S. Temir and A. Kiliçman. This is an open access article distributed under the Creative Commons Attribution License, which permits unrestricted use, distribution, and reproduction in any medium, provided the original work is properly cited.

Let $X$ be a real Banach space and $K$ a nonempty closed convex subset of $X$. Let $T_{i}: K \rightarrow K(i=1,2, \ldots, m)$ be $m$ asymptotically nonexpansive mappings with sequence $\left\{k_{n}\right\} \subset[1, \infty), \sum_{n=1}^{\infty}\left(k_{n}-1\right)<\infty$, and $\mathscr{F}=\bigcap_{i=1}^{m} F\left(T_{i}\right) \neq \emptyset$, where $F$ is the set of fixed points of $T_{i}$. Suppose that $\left\{a_{i n}\right\}_{n=1}^{\infty},\left\{b_{i n}\right\}_{n=1}^{\infty}, i=1,2, \ldots, m$ are appropriate sequences in $[0,1]$ and $\left\{u_{i n}\right\}_{n=1}^{\infty}, i=1,2, \ldots, m$ are bounded sequences in $K$ such that $\sum_{n=1}^{\infty} b_{i n}<\infty$ for $i=1,2, \ldots, m$. We give $\left\{x_{n}\right\}$ defined by $x_{1} \in K, x_{n+1}=\left(1-a_{1 n}-b_{1 n}\right) y_{n+m-2}+$ $a_{1 n} T_{1}^{n} y_{n+m-2}+b_{1 n} u_{1 n}, y_{n+m-2}=\left(1-a_{2 n}-b_{2 n}\right) y_{n+m-3}+a_{2 n} T_{2}^{n} y_{n+m-3}+b_{2 n} u_{2 n}, \ldots, y_{n+2}=\left(1-a_{(m-2) n}-b_{(m-2) n}\right) y_{n+1}+a_{(m-2) n} T_{m-2}^{n} y_{n+1}+$ $b_{(m-2) n} u_{(m-2) n}, y_{n+1}=\left(1-a_{(m-1) n}-b_{(m-1) n}\right) y_{n}+a_{(m-1) n} T_{m-1}^{n} y_{n}+b_{(m-1) n} u_{(m-1) n}, y_{n}=\left(1-a_{m n}-b_{m n}\right) x_{n}+a_{m n} T_{m}^{n} x_{n}+b_{m n} u_{m n}, m \geq 2, n \geq 1$. The purpose of this paper is to study the above iteration scheme for approximating common fixed points of a finite family of asymptotically nonexpansive mappings and to prove weak and some strong convergence theorems for such mappings in real Banach spaces. The results obtained in this paper extend and improve some results in the existing literature.

\section{Introduction}

Let $K$ be a nonempty subset of a real Banach space $X$ and let $T: K \rightarrow K$ be a mapping. Let $F(T)=\{x \in K: T x=x\}$ be the set of fixed points of $T$.

A mapping $T: K \rightarrow K$ is called nonexpansive if

$$
\|T x-T y\| \leq\|x-y\|
$$

for all $x, y \in K$. Similarly, $T$ is called asymptotically nonexpansive if there exists a sequence $\left\{k_{n}\right\} \subset[1, \infty)$ with $\lim _{n \rightarrow \infty} k_{n}=1$ such that

$$
\left\|T^{n} x-T^{n} y\right\| \leq k_{n}\|x-y\|
$$

for all $x, y \in K$ and $n \geq 1$. The mapping $T$ is called uniformly
L-Lipschitzian if there exists a positive constant $L$ such that

$$
\left\|T^{n} x-T^{n} y\right\| \leq L\|x-y\|
$$

for all $x, y \in K$ and $n \geq 1$.

It is easy to see that if $T$ is asymptotically nonexpansive, then it is uniformly $L$-Lipschitzian with the uniform Lipschitz constant $L=\sup \left\{k_{n}: n \geq 1\right\}$.

The class of asymptotically nonexpansive mappings which is an important generalization of the class nonexpansive maps was introduced by Goebel and Kirk [1]. They proved that every asymptotically nonexpansive self-mapping of a nonempty closed convex bounded subset of a uniformly convex Banach space has a fixed point.

The main tool for approximation of fixed points of generalizations of nonexpansive mappings remains iterative technique. Iterative techniques for nonexpansive selfmappings in Banach spaces including Mann type (one-step), Ishikawa type (two-step), and three-step iteration processes 
have been studied extensively by various authors; see, for example, $([2-8])$.

Recently, Chidume and Ali [9] defined (4) and constructed the sequence for the approximation of common fixed points of finite families of asymptotically nonexpansive mappings. Yıldırım and Özdemir [10] introduced an iteration scheme for approximating common fixed points of a finite family of asymptotically quasi-nonexpansive self-mappings and proved some strong and weak convergence theorems for such mappings in uniformly convex Banach spaces. Quan et al. [11] studied sufficient and necessary conditions for finite step iterative schemes with mean errors for a finite family of asymptotically quasi-nonexpansive mappings in Banach spaces to converge to a common fixed point. Peng [12] proved the convergence of finite step iterative schemes with mean errors for asymptotically nonexpansive mappings in Banach spaces. More recently Kızıltunç and Temir [13] introduced and studied a new iteration process for a finite family of nonself asymptotically nonexpansive mappings with errors in Banach spaces.

In [9], the authors introduced an iterative process for a finite family of asymptotically nonexpansive mappings as follows:

$$
\begin{aligned}
& x_{1} \in K, \\
& x_{n+1}=\left(1-a_{1 n}\right) x_{n}+a_{1 n} T_{1}^{n} y_{n+m-2}, \\
& y_{n+m-2}=\left(1-a_{2 n}\right) x_{n}+a_{2 n} T_{2}^{n} y_{n+m-3}, \\
& \vdots \\
& y_{n+1}=\left(1-a_{(m-1) n}\right) x_{n}+a_{(m-1) n} T_{m-1}^{n} y_{n}, \\
& y_{n}=\left(1-a_{m n}\right) x_{n}+a_{m n} T_{m}^{n} x_{n}, \quad \text { if } m \geq 2, n \geq 1,
\end{aligned}
$$

where $T_{1}, T_{2}, \ldots, T_{m}: K \rightarrow K$ are $m$ asymptotically nonexpansive mappings and $\left\{a_{i n}\right\} \subset[0,1]$ for $i=1, \ldots, m$.

Inspired and motivated by these facts, it is our purpose in this paper to construct an iteration scheme for approximating common fixed points of finite family of asymptotically nonexpansive mappings and study weak and some strong convergence theorems for such mappings in real Banach spaces.

Let $X$ be a real Banach space and $K$ a nonempty closed convex subset of $X$. Let $T_{i}: K \rightarrow K(i=1,2, \ldots, m)$ be $m$ asymptotically nonexpansive mappings with sequence $\left\{k_{n}\right\} \subset[1, \infty), \sum_{n=1}^{\infty}\left(k_{n}-1\right)<\infty$, and $\mathscr{F}=\bigcap_{i=1}^{m} F\left(T_{i}\right) \neq \emptyset$. Suppose that $\left\{a_{i n}\right\}_{n=1}^{\infty},\left\{b_{i n}\right\}_{n=1}^{\infty}, i=1,2, \ldots, m$ are appropriate sequences in $[0,1]$ and $\left\{u_{i n}\right\}_{n=1}^{\infty}, i=1,2, \ldots, m$ are bounded sequences in $K$ such that $\sum_{n=1}^{\infty} b_{i n}<\infty$ for $i=1,2, \ldots, m$. Let $\left\{x_{n}\right\}$ be defined by

$$
\begin{aligned}
x_{1} \in K & \\
x_{n+1}= & \left(1-a_{1 n}-b_{1 n}\right) y_{n+m-2}+a_{1 n} T_{1}^{n} y_{n+m-2}+b_{1 n} u_{1 n} \\
y_{n+m-2}= & \left(1-a_{2 n}-b_{2 n}\right) y_{n+m-3} \\
& +a_{2 n} T_{2}^{n} y_{n+m-3}+b_{2 n} u_{2 n}
\end{aligned}
$$

$\vdots$

$$
\begin{aligned}
& y_{n+2}=\left(1-a_{(m-2) n}-b_{(m-2) n}\right) y_{n+1} \\
&+a_{(m-2) n} T_{m-2}^{n} y_{n+1}+b_{(m-2) n} u_{(m-2) n}, \\
& y_{n+1}=\left(1-a_{(m-1) n}-b_{(m-1) n}\right) y_{n} \\
&+a_{(m-1) n} T_{m-1}^{n} y_{n}+b_{(m-1) n} u_{(m-1) n}, \\
& y_{n}=\left(1-a_{m n}-b_{m n}\right) x_{n}+a_{m n} T_{m}^{n} x_{n} \\
& \quad+b_{m n} u_{m n}, \quad m \geq 2, n \geq 1 .
\end{aligned}
$$

\section{Preliminaries}

Let $X$ be a real Banach space, $K$ a nonempty closed convex subset of $X$, and $F(T)$ the set of fixed points of $T$. A Banach space $X$ is said to be uniformly convex if the modulus of convexity of $X$

$$
\delta(\varepsilon)=\inf \left\{1-\frac{\|x+y\|}{2}:\|x\|=\|y\|=1,\|x-y\|=\varepsilon\right\}>0
$$

for all $0<\varepsilon \leq 2$ (i.e., $\delta:(0,2] \rightarrow[0,1])$. Recall that a Banach space $X$ is said to satisfy Opial's condition if, for each sequence $\left\{x_{n}\right\}$ in $X$, the condition $x_{n} \rightarrow x$ implies that

$$
\liminf _{n \rightarrow \infty}\left\|x_{n}-x\right\|<\liminf _{n \rightarrow \infty}\left\|x_{n}-y\right\|
$$

for all $y \in X$ with $y \neq x$. It is well known that all $l_{r}$ spaces for $1<r<\infty$ have this property. However, the $L_{r}$ spaces do not have unless $r=2$.

A mapping $T: K \rightarrow K$ is said to be semicompact if, for any bounded sequence $\left\{x_{n}\right\}$ in $K$ such that $\left\|x_{n}-T x_{n}\right\| \rightarrow 0$ as $n \rightarrow \infty$, there exists a subsequence say $\left\{x_{n_{j}}\right\}$ of $\left\{x_{n}\right\}$ such that $\left\{x_{n_{j}}\right\}$ converges strongly to some $x^{*}$ in $K . T$ is said to be completely continuous if for every bounded sequence $\left\{x_{n}\right\}$ in $K$, there exists a subsequence say $\left\{x_{n_{j}}\right\}$ of $\left\{x_{n}\right\}$ such that the sequence $\left\{T x_{n_{j}}\right\}$ converges strongly to some element of the range of $T$.

The following lemmas were given in $[14,15]$, respectively, and we need them to prove our main results.

Lemma 1. Let $\left\{s_{n}\right\},\left\{t_{n}\right\}$, and $\left\{\sigma_{n}\right\}$ be sequences of nonnegative real numbers satisfying the following conditions: for all $n \geq 1$, $s_{n+1} \leq\left(1+\sigma_{n}\right) s_{n}+t_{n}$, where $\sum_{n=1}^{\infty} \sigma_{n}<\infty$ and $\sum_{n=1}^{\infty} t_{n}<\infty$. Then

(i) $\lim _{n \rightarrow \infty} s_{n}$ exists;

(ii) in particular, if $\left\{s_{n}\right\}$ has a subsequence $\left\{s_{n_{j}}\right\}$ converging to 0 , then $\lim _{n \rightarrow \infty} s_{n}=0$.

Lemma 2. Let $p>1$ and $C>0$ be two fixed numbers. Then a Banach space $X$ is uniformly convex if and only if there exists a 
continuous, strictly increasing, convex function $g:[0, \infty) \rightarrow$ $[0, \infty)$ with $g(0)=0$ such that

$$
\begin{aligned}
\|\lambda x+(1-\lambda) y\|^{p} & \leq \lambda\|x\|^{p}+(1-\lambda)\|y\|^{p} \\
& -w_{p}(\lambda) g(\|x-y\|)
\end{aligned}
$$

for all $x, y \in B_{C}:=\{x \in X:\|x\| \leq C\}$, and $\lambda \in[0,1]$, where $w_{p}(\lambda)=\lambda(1-\lambda)^{p}+\lambda^{p}(1-\lambda)$.

The following lemmas were proved in [3].

Lemma 3. Let $X$ be a uniformly convex Banach space and $B_{C}:=\{x \in X:\|x\| \leq C\}, C>0$. Then there exists a continuous, strictly increasing, convex function $g:[0, \infty) \rightarrow$ $[0, \infty)$ with $g(0)=0$ such that

$$
\begin{aligned}
\|\lambda x+\mu y+\nu z\|^{2} \leq & \lambda\|x\|^{2}+\mu\|y\|^{2} \\
& +\nu\|z\|^{2}-(\lambda \mu) g(\|x-y\|)
\end{aligned}
$$

for all $x, y, z \in B_{C}$ and $\lambda, \mu, \nu \in[0,1]$ with $\lambda+\mu+\nu=1$.

Lemma 4. Let $X$ be a uniformly convex Banach space, $K a$ nonempty closed convex subset of $X$, and $T: K \rightarrow K$ an asymptotically nonexpansive mapping. Then $I-T$ ( $I$ is identity mapping) is demiclosed at zero; that is, if $x_{n} \rightarrow x^{*}$ weakly and $x_{n}-T x_{n} \rightarrow 0$ strongly, then $x^{*} \in F(T)$, where $F(T)$ is the set of fixed points of $T$.

Definition 5. A family $\left\{T_{i}: i \in\{1, \ldots, m\}\right\}$ of asymptotically nonexpansive mappings on $K$ with $\mathscr{F}=\bigcap_{i=1}^{m} F\left(T_{i}\right) \neq \emptyset$ is said to satisfy condition (A) on $K$ if there exists a nondecreasing function $f:[0, \infty) \rightarrow[0, \infty)$ with $f(0)=0, f(r)>0$, for all $r \in(0, \infty)$ such that $\max _{1 \leq i \leq m}\left\|x-T_{i} x\right\| \geq f(d(x, F))$ for all $x \in K$.

\section{Main Results}

In this section, we prove weak and strong convergence of the iterative sequence generated by iterative scheme (5) to a common element of the sets of fixed points of a finite family of asymptotically nonexpansive mappings in a real Banach space.

Lemma 6. Let $X$ be a real Banach space and $K$ a nonempty closed convex subset of $X$. Let $T_{i}: K \rightarrow K(i=1,2, \ldots, m)$ be $m$ asymptotically nonexpansive mappings with sequence $\left\{k_{n}\right\} \subset$ $[1, \infty), \sum_{n=1}^{\infty}\left(k_{n}-1\right)<\infty$, and $\mathscr{F}=\bigcap_{i=1}^{m} F\left(T_{i}\right) \neq \emptyset$. Suppose that $\left\{a_{i n}\right\}_{n=1}^{\infty},\left\{b_{i n}\right\}_{n=1}^{\infty}, i=1,2, \ldots, m$ are appropriate sequences in $[0,1]$ and $\left\{u_{i n}\right\}_{n=1}^{\infty}, i=1,2, \ldots, m$ are bounded sequences in $K$ such that $\sum_{n=1}^{\infty} b_{i n}<\infty$ for $i=1,2, \ldots, m$. Let $\left\{x_{n}\right\}$ be given by (5). Then $\left\{x_{n}\right\}$ is bounded and $\lim _{n \rightarrow \infty}\left\|x_{n}-p\right\|$ exists for $p \in \mathscr{F}$.

Proof. For any given $p \in \mathscr{F}$, since $\left\{u_{i n}\right\}_{n=1}^{\infty}, i=1,2, \ldots, m$ are bounded sequences in $K$, let

$$
M=\sup _{n \geq 1, i=1,2, \ldots, m}\left\|u_{i n}-p\right\| .
$$

For each $n \geq 1$, using (5), we have

$$
\begin{aligned}
& \left\|y_{n}-p\right\| \\
& =\left\|\left(\left(1-a_{m n}-b_{m n}\right) x_{n}+a_{m n} T_{m}^{n} x_{n}+b_{m n} u_{m n}\right)-p\right\| \\
& \leq\left(1-a_{m n}-b_{m n}\right)\left\|x_{n}-p\right\|+a_{m n}\left\|T_{m}^{n} x_{n}-p\right\| \\
& +b_{m n}\left\|u_{m n}-p\right\| \\
& \leq\left(1-a_{m n}-b_{m n}\right)\left\|x_{n}-p\right\|+a_{m n} k_{n}\left\|x_{n}-p\right\| \\
& +b_{m n}\left\|u_{m n}-p\right\| \\
& \leq k_{n}\left\|x_{n}-p\right\|+b_{m n} M, \\
& \left\|y_{n+1}-p\right\| \\
& =\|\left(\left(1-a_{(m-1) n}-b_{(m-1) n}\right) y_{n}+a_{(m-1) n} T_{(m-1)}^{n} y_{n}\right. \\
& \left.+b_{(m-1) n} u_{(m-1) n}\right)-p \| \\
& \leq\left(1-a_{(m-1) n}-b_{(m-1) n}\right)\left\|y_{n}-p\right\|+a_{(m-1) n} \\
& \times\left\|T_{(m-1)}^{n} y_{n}-p\right\|+b_{(m-1) n}\left\|u_{(m-1) n}-p\right\| \\
& \leq\left(1-a_{(m-1) n}-b_{(m-1) n}\right)\left\|y_{n}-p\right\|+a_{(m-1) n} k_{n} \\
& \times\left\|y_{n}-p\right\|+b_{(m-1) n}\left\|u_{(m-1) n}-p\right\| \\
& \leq k_{n}\left\|y_{n}-p\right\|+b_{(m-1) n} M \\
& \leq k_{n}^{2}\left\|x_{n}-p\right\|+b_{m n} M k_{n}+b_{(m-1) n} M, \\
& \left\|y_{n+2}-p\right\| \\
& =\|\left(\left(1-a_{(m-2) n}-b_{(m-2) n}\right) y_{n+1}+a_{(m-2) n} T_{(m-2)}^{n} y_{n+1}\right. \\
& \left.+b_{(m-2) n} u_{(m-2) n}\right)-p \| \\
& \leq\left(1-a_{(m-2) n}-b_{(m-2) n}\right)\left\|y_{n+1}-p\right\| \\
& +a_{(m-2) n}\left\|T_{(m-2)}^{n} y_{n+1}-p\right\| \\
& +b_{(m-2) n}\left\|u_{(m-2) n}-p\right\| \\
& \leq\left(1-a_{(m-2) n}-b_{(m-2) n}\right)\left\|y_{n+1}-p\right\| \\
& +a_{(m-2) n} k_{n}\left\|y_{n+1}-p\right\| \\
& +b_{(m-2) n}\left\|u_{(m-2) n}-p\right\| \\
& \leq k_{n}\left\|y_{n+1}-p\right\|+b_{(m-2) n} M \\
& \leq k_{n}^{3}\left\|x_{n}-p\right\|+b_{m n} M k_{n}^{2}+k_{n} b_{(m-1) n} M+b_{(m-2) n} M, \\
& \left\|y_{n+m-2}-p\right\| \\
& =\left\|\left(\left(1-a_{2 n}-b_{2 n}\right) y_{n+m-3}+a_{2 n} T_{2}^{n} y_{n+m-3}+b_{2 n} u_{2 n}\right)-p\right\| \\
& \leq\left(1-a_{2 n}-b_{2 n}\right)\left\|y_{n+m-3}-p\right\|+a_{2 n}
\end{aligned}
$$




$$
\begin{aligned}
& \times\left\|T_{2}^{n} y_{n+m-3}-p\right\|+b_{2 n}\left\|u_{2 n}-p\right\| \\
\leq & \left(1-a_{2 n}-b_{2 n}\right)\left\|y_{n+m-3}-p\right\|+a_{2 n} k_{n} \\
& \times\left\|y_{n+m-3}-p\right\|+b_{2 n}\left\|u_{2 n}-p\right\| \\
\leq & k_{n}\left\|y_{n+m-3}-p\right\|+b_{2 n} M \\
\leq & k_{n}^{(m-1)}\left\|x_{n}-p\right\|+k_{n}^{(m-2)} b_{m n} M+k_{n}^{(m-3)} b_{(m-1) n} M \\
& +\cdots+k_{n} b_{3 n} M+b_{2 n} M, \\
\| x_{n+1} & -p \| \\
= & \left\|\left(\left(1-a_{1 n}-b_{1 n}\right) y_{n+m-2}+a_{1 n} T_{1}^{n} y_{n+m-2}+b_{1 n} u_{1 n}\right)-p\right\| \\
\leq & \left(1-a_{1 n}-b_{1 n}\right)\left\|y_{n+m-2}-p\right\|+a_{1 n} \\
& \times\left\|T_{1}^{n} y_{n+m-2}-p\right\|+b_{1 n}\left\|u_{1 n}-p\right\| \\
\leq & \left(1-a_{1 n}-b_{1 n}\right)\left\|y_{n+m-2}-p\right\|+a_{1 n} k_{n} \\
& \times\left\|y_{n+m-2}-p\right\|+b_{1 n}\left\|u_{1 n}-p\right\| \\
\leq & k_{n}\left\|y_{n+m-2}-p\right\|+b_{1 n} M \\
\leq & k_{n}^{m}\left\|x_{n}-p\right\|+k_{n}^{(m-1)} b_{m n} M+k_{n}^{(m-2)} b_{(m-1) n} M \\
& +\cdots+k_{n}^{2} b_{3 n} M+k_{n} b_{2 n} M+b_{1 n} M .
\end{aligned}
$$

Then we have

$$
\left\|x_{n+1}-p\right\| \leq k_{n}^{m}\left\|x_{n}-p\right\|+M \sum_{i=1}^{m} k_{n}^{(i-1)} b_{i n}
$$

which leads to

$$
\left\|x_{n+1}-p\right\| \leq\left(1+\left(k_{n}^{m}-1\right)\right)\left\|x_{n}-p\right\|+\varphi_{n}, \quad n \geq 1,
$$

where

$$
\varphi_{n}=M \sum_{i=1}^{m} k_{n}^{(i-1)} b_{i n}
$$

Since $t^{m}-1 \leq m t^{m-1}(t-1)$ for all $t \geq 1$, the only assumption $\sum_{n=1}^{\infty}\left(k_{n}-1\right)<\infty$ is enough for the boundedness for $\left\{k_{n}\right\}$, then $k_{n} \subset[1, D]$, for all $n \geq 1$, and for some $D$. Hence $k_{n}^{m}-1 \leq$ $m D^{m-1}\left(k_{n}-1\right)$ holds for all $n \geq 1$. Therefore $\sum_{n=1}^{\infty}\left(k_{n}^{m}-1\right)<\infty$ and also $\sum_{n=1}^{\infty} \varphi_{n}<\infty$. Equation (13) and Lemma 1 guarantee that the sequence $\left\{x_{n}\right\}$ is bounded and $\lim _{n \rightarrow \infty}\left\|x_{n}-p\right\|$ exists.

Theorem 7. Let $X$ be a real uniformly convex Banach space and $K$ a nonempty closed convex subset of $X$. Let $T_{i}$ : $K \rightarrow K(i=1,2, \ldots, m)$ be $m$ asymptotically nonexpansive mappings with sequence $\left\{k_{n}\right\} \subset[1, \infty), \sum_{n=1}^{\infty}\left(k_{n}-1\right)<\infty$ and $\mathscr{F}=\bigcap_{i=1}^{m} F\left(T_{i}\right) \neq \emptyset$. Suppose that $\left\{a_{i n}\right\}_{n=1}^{\infty},\left\{b_{i n}\right\}_{n=1}^{\infty}, i=$ $1,2, \ldots, m$ are appropriate sequences in $[0,1]$ and $\left\{u_{i n}\right\}_{n=1}^{\infty}, i=$ $1,2, \ldots, m$ are bounded sequences in $K$ such that $\sum_{n=1}^{\infty} b_{i n}<\infty$ for $i=1,2, \ldots, m$. Let $\left\{x_{n}\right\}$ be given by (5). Suppose that

$$
0<\liminf _{n \rightarrow \infty} a_{i n}<\limsup _{n \rightarrow \infty}\left(a_{i n}+b_{i n}\right)<1
$$

for $i=1, \ldots, m$. Then

$$
\lim _{n \rightarrow \infty}\left\|T_{i} x_{n}-x_{n}\right\|=0, \quad(i=1,2, \ldots, m) .
$$

Proof. Let $p \in \mathscr{F}$. Then by Lemma $6, \lim _{n \rightarrow \infty}\left\|x_{n}-p\right\|$ exists. Since $\left\{u_{i n}\right\}_{n=1}^{\infty}, i=1,2, \ldots, m$ are bounded sequences in $K$, let $M=\sup _{n \geq 1, i=1,2, \ldots, m}\left\|u_{\text {in }}-p\right\|$; moreover, it follows that $\left\{y_{n+m-i}-p\right\}$ is also bounded for each $i \in\{2,3, \ldots, m\}$, and hence $\left\{\left(u_{(m-i+1) n}-y_{n+i-1}\right)\right\}$ is also bounded for $i \in$ $\{1,2, \ldots, m\}$. By using (5), we obtain

$$
\begin{aligned}
& \left\|y_{n}-p\right\|^{2} \\
& =\left\|\left(\left(1-a_{m n}-b_{m n}\right) x_{n}+a_{m n} T_{m}^{n} x_{n}+b_{m n} u_{m n}\right)-p\right\|^{2} \\
& \leq\left(1-a_{m n}-b_{m n}\right)\left\|x_{n}-p\right\|^{2}+a_{m n}\left\|T_{m}^{n} x_{n}-p\right\|^{2} \\
& +b_{m n}\left\|u_{m n}-p\right\|^{2} \\
& -\left(1-a_{m n}-b_{m n}\right) a_{m n} g\left(\left\|T_{m}^{n} x_{n}-x_{n}\right\|\right) \\
& \leq\left(1-a_{m n}-b_{m n}\right)\left\|x_{n}-p\right\|^{2}+a_{m n} k_{n}^{2} \\
& \times\left\|x_{n}-p\right\|^{2}+b_{m n}\left\|u_{n m}-p\right\|^{2} \\
& -\left(1-a_{m n}-b_{m n}\right) a_{m n} g\left(\left\|T_{m}^{n} x_{n}-x_{n}\right\|\right) \\
& \leq k_{n}^{2}\left\|x_{n}-p\right\|^{2}+b_{m n} M^{2}-\left(1-a_{m n}-b_{m n}\right) \\
& \times a_{m n} g\left(\left\|T_{m}^{n} x_{n}-x_{n}\right\|\right) \text {, } \\
& \left\|y_{n+1}-p\right\|^{2} \\
& =\|\left(\left(1-a_{(m-1) n}-b_{(m-1) n}\right) y_{n}+a_{(m-1) n}\right. \\
& \left.\times T_{(m-1)}^{n} y_{n}+b_{(m-1) n} u_{(m-1) n}\right)-p \|^{2} \\
& \leq\left(1-a_{(m-1) n}-b_{(m-1) n}\right)\left\|y_{n}-p\right\|^{2} \\
& +a_{(m-1) n}\left\|T_{(m-1)}^{n} y_{n}-p\right\|^{2}+b_{(m-1) n}\left\|u_{(m-1) n}-p\right\|^{2} \\
& -\left(1-a_{(m-1) n}-b_{(m-1) n}\right) a_{(m-1) n} g\left(\left\|T_{(m-1)}^{n} y_{n}-y_{n}\right\|\right) \\
& \leq\left(1-a_{(m-1) n}-b_{(m-1) n}\right)\left\|y_{n}-p\right\|^{2} \\
& +a_{(m-1) n} k_{n}^{2}\left\|y_{n}-p\right\|^{2}+b_{(m-1) n} M^{2} \\
& -\left(1-a_{(m-1) n}-b_{(m-1) n}\right) a_{(m-1) n} g\left(\left\|T_{(m-1)}^{n} y_{n}-y_{n}\right\|\right) \\
& \leq k_{n}^{2}\left\|y_{n}-p\right\|^{2}+b_{(m-1) n} M^{2}-\left(1-a_{(m-1) n}-b_{(m-1) n}\right) \\
& \times a_{(m-1) n} g\left(\left\|T_{(m-1)}^{n} y_{n}-y_{n}\right\|\right) \\
& \leq k_{n}^{2}\left(k_{n}^{2}\left\|x_{n}-p\right\|^{2}+b_{m n} M^{2}-\left(1-a_{m n}-b_{m n}\right)\right. \\
& \left.\times a_{m n} g\left(\left\|T_{m}^{n} x_{n}-x_{n}\right\|\right)\right)+b_{(m-1) n} M^{2} \\
& -\left(1-a_{(m-1) n}-b_{(m-1) n}\right) a_{(m-1) n} g\left(\left\|T_{(m-1)}^{n} y_{n}-y_{n}\right\|\right) \text {, }
\end{aligned}
$$




$$
\begin{aligned}
\| y_{n+1} & -p \|^{2} \\
\leq & k_{n}^{4}\left\|x_{n}-p\right\|^{2}+k_{n}^{2} b_{m n} M^{2}+b_{(m-1) n} M^{2} \\
& -\left(1-a_{m n}-b_{m n}\right) a_{m n} g\left(\left\|T_{m}^{n} x_{n}-x_{n}\right\|\right) \\
& -\left(1-a_{(m-1) n}-b_{(m-1) n}\right) a_{(m-1) n} g\left(\left\|T_{(m-1)}^{n} y_{n}-y_{n}\right\|\right),
\end{aligned}
$$$$
\left\|y_{n+2}-p\right\|^{2}
$$$$
=\|\left(\left(1-a_{(m-2) n}-b_{(m-2) n}\right) y_{n+1}+a_{(m-2) n} T_{(m-2)}^{n} y_{n+1}\right.
$$$$
\left.+b_{(m-2) n} u_{(m-2) n}\right)-p \|^{2}
$$$$
\leq\left(1-a_{(m-2) n}-b_{(m-2) n}\right)\left\|y_{n+1}-p\right\|^{2}
$$$$
+a_{(m-2) n}\left\|T_{(m-2)}^{n} y_{n+1}-p\right\|^{2}
$$$$
+b_{(m-2) n}\left\|u_{(m-2) n}-p\right\|^{2}
$$$$
-\left(1-a_{(m-2) n}-b_{(m-2) n}\right) a_{(m-2) n}
$$$$
\times g\left(\left\|T_{(m-2)}^{n} y_{n+1}-y_{n+1}\right\|\right)
$$$$
\leq\left(1-a_{(m-2) n}-b_{(m-2) n}\right)\left\|y_{n+1}-p\right\|^{2}
$$$$
+a_{(m-2) n} k_{n}^{2}\left\|y_{n+1}-p\right\|^{2}+b_{(m-2) n} M^{2}
$$$$
-\left(1-a_{(m-2) n}-b_{(m-2) n}\right) a_{(m-2) n}
$$$$
\times g\left(\left\|T_{(m-2)}^{n} y_{n+1}-y_{n+1}\right\|\right)
$$$$
\leq k_{n}^{2}\left\|y_{n+1}-p\right\|^{2}+b_{(m-2) n} M^{2}
$$$$
-\left(1-a_{(m-2) n}-b_{(m-2) n}\right) a_{(m-2) n}
$$$$
\times g\left(\left\|T_{(m-2)}^{n} y_{n+1}-y_{n+1}\right\|\right)
$$$$
\leq k_{n}^{2}\left(k_{n}^{4}\left\|x_{n}-p\right\|^{2}+k_{n}^{2} b_{m n} M^{2}+b_{(m-1) n} M^{2}\right.
$$$$
-\left(1-a_{m n}-b_{m n}\right) a_{m n} g\left(\left\|T_{m}^{n} x_{n}-x_{n}\right\|\right)
$$$$
-\left(1-a_{(m-1) n}-b_{(m-1) n}\right) a_{(m-1) n}
$$$$
\left.\times g\left(\left\|T_{(m-1)}^{n} y_{n}-y_{n}\right\|\right)\right)+b_{(m-2) n} M^{2}
$$$$
-\left(1-a_{(m-2) n}-b_{(m-2) n}\right) a_{(m-2) n}
$$$$
\times g\left(\left\|T_{(m-2)}^{n} y_{n+1}-y_{n+1}\right\|\right)
$$$$
\leq k_{n}^{6}\left\|x_{n}-p\right\|^{2}+k_{n}^{4} b_{m n} M^{2}+k_{n}^{2} b_{(m-1) n} M^{2}+b_{(m-2) n} M^{2}
$$$$
-\left(1-a_{m n}-b_{m n}\right) a_{m n} g\left(\left\|T_{m}^{n} x_{n}-x_{n}\right\|\right)
$$$$
-\left(1-a_{(m-1) n}-b_{(m-1) n}\right) a_{(m-1) n} g\left(\left\|T_{(m-1)}^{n} y_{n}-y_{n}\right\|\right)
$$$$
-\left(1-a_{(m-2) n}-b_{(m-2) n}\right) a_{(m-2) n}
$$$$
\times g\left(\left\|T_{(m-2)}^{n} y_{n+1}-y_{n+1}\right\|\right)
$$

$$
\begin{aligned}
& \left\|x_{n+1}-p\right\|^{2} \\
& \leq k_{n}^{2 m}\left\|x_{n}-p\right\|^{2}+M^{2} \sum_{i=0}^{m-1} k_{n}^{2 i} b_{(i+1) n} \\
& \quad-\sum_{i=0}^{m-2}\left(\left(1-a_{(i+1) n}-b_{(i+1) n}\right) a_{(i+1) n}\right. \\
& \left.\quad \times g\left(\left\|T_{i+1}^{n} y_{n+m-i-2}-y_{n+m-i-2}\right\|\right)\right) \\
& -\left(1-a_{m n}-b_{m n}\right) a_{m n} g\left(\left\|T_{m}^{n} x_{n}-x_{n}\right\|\right) .
\end{aligned}
$$

Note that $0 \leq \theta^{2}-1 \leq 2 \theta(\theta-1)$ for all $\theta \geq 1$, the assumption $\sum_{n=1}^{\infty}\left(k_{n}-1\right)<\infty$ implies that $\sum_{n=1}^{\infty}\left(k_{n}^{2}-1\right)<\infty$. Since $\left\{k_{n}\right\}$ is bounded, there exists $D>0$ such that $k_{n} \in[1, D], n \geq 1$. Then $k_{n}^{2 m}-1 \leq 2 m D^{2 m-1}\left(k_{n}-1\right)$ holds for all $n \geq 1$. Therefore, the assumption $\sum_{n=1}^{\infty}\left(k_{n}-1\right)<\infty$ implies that $\sum_{n=1}^{\infty}\left(k_{n}^{2 m}-1\right)<\infty$. Then

$$
\left(k_{n}^{2 m}-1\right)\left\|x_{n}-p\right\|^{2} \leq 2 m D^{2 m-1} C^{2}\left(k_{n}-1\right) .
$$

It follows from (18) and (19) that

$$
\begin{aligned}
& \left(1-a_{m n}-b_{m n}\right) a_{m n} g\left(\left\|T_{m}^{n} x_{n}-x_{n}\right\|\right) \\
& +\sum_{i=0}^{m-1}\left(\left(1-a_{(i+1) n}-b_{(i+1) n}\right) a_{(i+1) n}\right. \\
& \left.\quad \times g\left(\left\|T_{i+1}^{n} y_{n+m-i-2}-y_{n+m-i-2}\right\|\right)\right) \\
& \leq\left\|x_{n}-p\right\|^{2}-\left\|x_{n+1}-p\right\|^{2}+2 m D^{2 m-1} C^{2}\left(k_{n}-1\right) \\
& +M^{2} \sum_{i=0}^{m-1} k_{n}^{2 i} b_{(i+1) n} .
\end{aligned}
$$

We first obtain that

$$
\begin{aligned}
& \left(1-a_{m n}-b_{m n}\right) a_{m n} g\left(\left\|T_{m}^{n} x_{n}-x_{n}\right\|\right) \\
& \leq\left\|x_{n}-p\right\|^{2}-\left\|x_{n+1}-p\right\|^{2}+2 m D^{2 m-1} C^{2}\left(k_{n}-1\right) \\
& \quad+M^{2} \sum_{i=0}^{m-1} k_{n}^{2 i} b_{(i+1) n} .
\end{aligned}
$$

Now if $0<\lim \inf _{n \rightarrow \infty} a_{m n}$ and $0<\lim \inf _{n \rightarrow \infty} a_{m n}<$ $\lim \sup _{n \rightarrow \infty}\left(a_{m n}+b_{m n}\right)<1$, there exist a positive integer $n_{0}$ and $\eta, \eta^{\prime} \in(0,1)$ such that $0<\eta<a_{m n}, a_{m n}+b_{m n}<\eta^{\prime}<1$ for all $n \geq n_{0}$. This implies by (21) that

$$
\begin{aligned}
& \eta\left(1-\eta^{\prime}\right) g\left(\left\|T_{m}^{n} x_{n}-x_{n}\right\|\right) \\
& \leq\left\|x_{n}-p\right\|^{2}-\left\|x_{n+1}-p\right\|^{2} \\
&+2 m D^{2 m-1} C^{2}\left(k_{n}-1\right)+M^{2} \sum_{i=0}^{m-1} k_{n}^{2 i} b_{(i+1) n} .
\end{aligned}
$$


It follows from (22) that for $\ell \geq n_{0}$,

$$
\begin{aligned}
& \sum_{n=n_{0}}^{\ell} g\left(\left\|T_{m}^{n} x_{n}-x_{n}\right\|\right) \\
& \leq \frac{1}{\eta\left(1-\eta^{\prime}\right)}\left(\sum_{n=n_{0}}^{\ell}\left(\left\|x_{n}-p\right\|^{2}-\left\|x_{n+1}-p\right\|^{2}\right)\right. \\
& +2 m D^{2 m-1} C^{2} \sum_{n=n_{0}}^{\ell}\left(k_{n}-1\right) \\
& \left.+M^{2} \sum_{n=n_{0}}^{\ell} \sum_{i=0}^{m-1} k_{n}^{2 i} b_{(i+1) n}\right) .
\end{aligned}
$$

Then $\sum_{n=n_{0}}^{\infty} g\left(\left\|T_{m}^{n} x_{n}-x_{n}\right\|\right)<\infty$, and therefore $\lim _{n \rightarrow \infty} g\left(\left\|T_{m}^{n} x_{n}-x_{n}\right\|\right)=0$, and by property of $g$, we have $\lim _{n \rightarrow \infty}\left\|T_{m}^{n} x_{n}-x_{n}\right\|=0$. By a similar method, together with (20) and by property of $g$, we have

$$
\begin{aligned}
\lim _{n \rightarrow \infty} & \left\|T_{m}^{n} x_{n}-x_{n}\right\| \\
& =\lim _{n \rightarrow \infty}\left\|T_{m-1}^{n} y_{n}-y_{n}\right\| \\
& =\lim _{n \rightarrow \infty}\left\|T_{m-2}^{n} y_{n+1}-y_{n+1}\right\| \\
\vdots & \\
= & \lim _{n \rightarrow \infty}\left\|T_{i}^{n} y_{n+m-i-1}-y_{n+m-i-1}\right\| \\
\vdots \quad & \\
& =\lim _{n \rightarrow \infty}\left\|T_{1}^{n} y_{n+m-2}-y_{n+m-2}\right\|=0
\end{aligned}
$$

for $2 \leq i<m$. Thus, we conclude that

$$
\limsup _{n \rightarrow \infty}\left\|T_{i-1}^{n} y_{n+m-i}-y_{n+m-i}\right\|=0
$$

for $2 \leq i \leq m$. From (5) and for $i=1,2, \ldots, m$

$$
\begin{aligned}
& \left\|y_{n+i}-y_{n+i-1}\right\| \\
& =\|\left(1-a_{(m-i) n}-b_{(m-i) n}\right) y_{n+i-1} \\
& \quad+a_{(m-i) n} T_{(m-i)}^{n} y_{n+i-1}+b_{(m-i) n} u_{(m-i) n}-y_{n+i-1} \| \\
& =\| a_{(m-i) n}\left(T_{(m-i)}^{n} y_{n+i-1}-y_{n+i-1}\right) \\
& \quad+b_{(m-i) n}\left(u_{(m-i) n}-y_{n+i-1}\right) \| \\
& \leq a_{(m-i) n}\left\|T_{(m-i)}^{n} y_{n+i-1}-y_{n+i-1}\right\| \\
& \quad+b_{(m-i) n}\left\|u_{(m-i) n}-y_{n+i-1}\right\| .
\end{aligned}
$$

This together with (25) implies that for each $i=1,2, \ldots, m-2$

$$
\lim _{n \rightarrow \infty}\left\|y_{n+i}-y_{n+i-1}\right\|=0 \text {. }
$$

It follows from (5) that

$$
\begin{aligned}
& \left\|y_{n}-x_{n}\right\| \\
& \quad=\left\|\left(1-a_{m n}-b_{m n}\right) x_{n}+a_{m n} T_{m}^{n} x_{n}+b_{m n} u_{m n}-x_{n}\right\| \\
& \quad \leq a_{m n}\left\|T_{m}^{n} x_{n}-x_{n}\right\|+b_{m n}\left\|u_{m n}-x_{n}\right\| .
\end{aligned}
$$

Equations (24) and (28) imply that

$$
\lim _{n \rightarrow \infty}\left\|y_{n}-x_{n}\right\|=0
$$

It follows from (5) that

$$
\begin{aligned}
& \left\|x_{n+1}-y_{n+m-2}\right\| \\
& =\|\left(1-a_{1 n}-b_{1 n}\right) y_{n+m-2}+a_{1 n} T_{1}^{n} y_{n+m-2} \\
& \quad \quad+b_{1 n} u_{1 n}-y_{n+m-2} \| \\
& \leq a_{1 n}\left\|T_{1}^{n} y_{n+m-2}-y_{n+m-2}\right\|+b_{1 n}\left\|u_{1 n}-y_{n+m-2}\right\| .
\end{aligned}
$$

Thus, (24) and (30) guarantee that

$$
\lim _{n \rightarrow \infty}\left\|x_{n+1}-y_{n+m-2}\right\|=0
$$

Continuing in this fashion, for each $i=2, \ldots, m$ we get,

$$
\begin{aligned}
& \lim _{n \rightarrow \infty}\left\|x_{n+1}-y_{n+i-2}\right\|=0, \\
& \left\|x_{n+1}-x_{n}\right\| \\
& =\left\|x_{n+1}-y_{n+m-2}+y_{n+m-2}-\cdots+y_{n+1}-y_{n}+y_{n}-x_{n}\right\| \\
& \leq\left\|x_{n+1}-y_{n+m-2}\right\|+\left\|y_{n+m-2}-y_{n+m-3}\right\| \\
& +\cdots+\left\|y_{n+1}-y_{n}\right\|+\left\|y_{n}-x_{n}\right\| .
\end{aligned}
$$

Taking the limit on both sides inequality from (33), we have

$$
\lim _{n \rightarrow \infty}\left\|x_{n+1}-x_{n}\right\|=0
$$

Since $T_{m}$ is an asymptotically nonexpansive mapping with $k_{n}$, we have

$$
\begin{aligned}
& \left\|x_{n+1}-T_{m}^{n} x_{n+1}\right\| \\
& \quad=\left\|x_{n+1}-x_{n}+x_{n}-T_{m}^{n} x_{n}+T_{m}^{n} x_{n}-T_{m}^{n} x_{n+1}\right\| \\
& \quad \leq\left\|x_{n+1}-x_{n}\right\|+\left\|T_{m}^{n} x_{n+1}-T_{m}^{n} x_{n}\right\|+\left\|T_{m}^{n} x_{n}-x_{n}\right\| \\
& \quad \leq\left\|x_{n+1}-x_{n}\right\|+k_{n}\left\|x_{n+1}-x_{n}\right\|+\left\|T_{m}^{n} x_{n}-x_{n}\right\| .
\end{aligned}
$$

Taking the limit on both sides inequality (35), and by using (24), we get

$$
\lim _{n \rightarrow \infty}\left\|x_{n+1}-T_{m}^{n} x_{n+1}\right\|=0 .
$$


Since $T_{m-1}$ is an asymptotically nonexpansive mapping with $k_{n}$, we have

$$
\begin{aligned}
& \left\|x_{n+1}-T_{m-1}^{n} x_{n+1}\right\| \\
& =\left\|x_{n+1}-y_{n}+y_{n}-T_{m-1}^{n} y_{n}+T_{m-1}^{n} y_{n}-T_{m-1}^{n} x_{n+1}\right\| \\
& \leq\left\|x_{n+1}-y_{n}\right\|+\left\|T_{m-1}^{n} x_{n+1}-T_{m-1}^{n} y_{n}\right\| \\
& \quad+\left\|T_{m-1}^{n} y_{n}-y_{n}\right\| \\
& \leq\left\|x_{n+1}-y_{n}\right\|+k_{n}\left\|x_{n+1}-y_{n}\right\|+\left\|T_{m-1}^{n} y_{n}-y_{n}\right\| .
\end{aligned}
$$

Also, taking the limit on both sides inequality (37), and by using (24), we get

$$
\lim _{n \rightarrow \infty}\left\|x_{n+1}-T_{m-1}^{n} x_{n+1}\right\|=0
$$

In a similar way, one can prove that for each $i=2, \ldots, m-1$

$$
\lim _{n \rightarrow \infty}\left\|x_{n}-T_{m-i}^{n} x_{n}\right\|=0 .
$$

Next, we consider

$$
\begin{aligned}
& \left\|x_{n}-T_{m} x_{n}\right\| \\
& \leq\left\|x_{n}-x_{n+1}\right\|+\left\|T_{m}^{n+1} x_{n+1}-x_{n+1}\right\| \\
& \quad+\left\|T_{m}^{n+1} x_{n+1}-T_{m}^{n+1} x_{n}\right\|+\left\|T_{m}^{n+1} x_{n}-T_{m} x_{n}\right\| \\
& \leq\left\|x_{n}-x_{n+1}\right\|+\left\|T_{m}^{n+1} x_{n+1}-x_{n+1}\right\| \\
& \quad+L\left\|x_{n+1}-x_{n}\right\|+L\left\|T_{m}^{n} x_{n}-x_{n}\right\| .
\end{aligned}
$$

It follows from (34), (36), and the above inequality (40) that

$$
\lim _{n \rightarrow \infty}\left\|x_{n}-T_{m} x_{n}\right\|=0
$$

$$
\begin{aligned}
& \left\|x_{n}-T_{m-1} x_{n}\right\| \\
& \leq\left\|x_{n}-x_{n+1}\right\|+\left\|T_{m-1}^{n+1} x_{n+1}-x_{n+1}\right\| \\
& \quad+\left\|T_{m-1}^{n+1} x_{n+1}-T_{m-1}^{n+1} x_{n}\right\|+\left\|T_{m-1}^{n+1} x_{n}-T_{m-1} x_{n}\right\| \\
& \leq\left\|x_{n}-x_{n+1}\right\|+\left\|T_{m-1}^{n+1} x_{n+1}-x_{n+1}\right\| \\
& \quad+L\left\|x_{n+1}-x_{n}\right\|+L\left\|T_{m-1}^{n} x_{n}-x_{n}\right\| .
\end{aligned}
$$

It follows from (34), (38) and (42) that

$$
\lim _{n \rightarrow \infty}\left\|x_{n}-T_{m-1} x_{n}\right\|=0 .
$$

Continuing similar process, for each $i=0, \ldots, m-1$ we get

$$
\lim _{n \rightarrow \infty}\left\|x_{n}-T_{m-i} x_{n}\right\|=0 .
$$

The proof is completed.

Theorem 8. Let $X$ be a real uniformly convex Banach space and $K$ a nonempty closed convex subset of $X$. Let $T_{i}$ : $K \rightarrow K(i=1,2, \ldots, m)$ be $m$ asymptotically nonexpansive mappings with sequence $\left\{k_{n}\right\} \subset[1, \infty), \sum_{n=1}^{\infty}\left(k_{n}-1\right)<\infty$ and $\mathscr{F}=\bigcap_{i=1}^{m} F\left(T_{i}\right) \neq \emptyset$. Suppose that $\left\{a_{i n}\right\}_{n=1}^{\infty},\left\{b_{i n}\right\}_{n=1}^{\infty}, i=$ $1,2, \ldots, m$ are appropriate sequences in $[0,1]$ and $\left\{u_{i n}\right\}_{n=1}^{\infty}, i=$ $1,2, \ldots, m$ are bounded sequences in $K$ such that $\sum_{n=1}^{\infty} b_{i n}<\infty$ for $i=1,2, \ldots, m$. Suppose that

$$
0<\liminf _{n \rightarrow \infty} a_{i n}<\lim _{n \rightarrow \infty} \sup _{n \rightarrow \infty}\left(a_{i n}+b_{i n}\right)<1
$$

for $i=1, \ldots, m$. If one of $\left\{T_{i}\right\}$ is either completely continuous or semicompact, for some $i \in\{1,2, \ldots, m\}$, then the sequence $\left\{x_{n}\right\}$ generated by (5) converges strongly to an element of $\mathscr{F}$.

Proof. Assume that there exists $\ell \in\{1,2, \ldots, m\}$ such that $T_{\ell}$ is semi-compact. Since $\left\{x_{n}\right\}$ is bounded and by Theorem 7 , $\left\|x_{n}-T_{\ell} x_{n}\right\| \rightarrow 0$ as $n \rightarrow \infty$, there exists a subsequence $\left\{x_{n_{j}}\right\}$ of $\left\{x_{n}\right\}$ such that $x_{n_{j}}$ converges strongly to $p \in K$. Since $\lim _{j \rightarrow \infty}\left\|x_{n_{j}}-T_{\ell} x_{n_{j}}\right\|=0$, it follows from Lemma 4 that $T_{\ell} p=p$. Also, from Theorem $7 \lim _{j \rightarrow \infty}\left\|x_{n_{j}}-T_{i} x_{n_{j}}\right\|=0$, $i=1,2, \ldots, m$. Therefore, from Lemma 4 we obtain that $p \in \bigcap_{i=1}^{m} F\left(T_{i}\right)$. So $\left\{x_{n}\right\}$ converges strongly to $p$.

If one of $T_{i}$ 's is completely continuous, say $T_{\ell}$, since $\left\{x_{n}\right\}$ is bounded, there exists a subsequence $\left\{x_{n_{j}}\right\}$ of $\left\{x_{n}\right\}$ such that $T_{\ell} x_{n_{j}}$ converges strongly to $p \in K$. By Theorem 7, $\lim _{j \rightarrow \infty}\left\|x_{n_{j}}-T_{\ell} x_{n_{j}}\right\|=0$. It follows from continuity of $\|\cdot\|$ that

$$
0=\lim _{j \rightarrow \infty}\left\|x_{n_{j}}-T_{\ell} x_{n_{j}}\right\|=\lim _{j \rightarrow \infty}\left\|x_{n_{j}}-p\right\|=0
$$

Using $\left\{x_{n_{j}}\right\} \rightarrow p$ as $j \rightarrow \infty, \lim _{j \rightarrow \infty}\left\|x_{n_{j}}-T_{i} x_{n_{j}}\right\|=0, i=$ $1,2, \ldots, m$ and Lemma 4 , we obtain that $p \in \mathscr{F}=\bigcap_{i=1}^{m} F\left(T_{i}\right)$. Also using $\left\{x_{n_{j}}\right\} \rightarrow p$ as $j \rightarrow \infty$ and Lemma 6, we obtain that $\lim _{n \rightarrow \infty}\left\|x_{n}-p\right\|=0$. This completes the proof.

Next, we prove a strong convergence theorem for asymptotically nonexpansive mappings in a uniformly convex Banach space satisfying condition (A).

Theorem 9. Let $X$ be a real uniformly convex Banach space and $K$ a nonempty closed convex subset of $X$. Let $T_{i}$ : $K \rightarrow K(i=1,2, \ldots, m)$ be $m$ asymptotically nonexpansive mappings with sequence $\left\{k_{n}\right\} \subset[1, \infty), \sum_{n=1}^{\infty}\left(k_{n}-1\right)<\infty$ and satisfying the condition (A). Suppose that $\left\{a_{i n}\right\}_{n=1}^{\infty},\left\{b_{i n}\right\}_{n=1}^{\infty}$, $i=1,2, \ldots, m$ are appropriate sequences in $[0,1]$ and $\left\{u_{i n}\right\}_{n=1}^{\infty}$, $i=1,2, \ldots, m$ are bounded sequences in K such that $\sum_{n=1}^{\infty} b_{i n}<$ $\infty$ for $i=1,2, \ldots, m$. Suppose that $\mathscr{F}=\bigcap_{i=1}^{m} F\left(T_{i}\right) \neq \emptyset$ and $0<\lim \inf _{n \rightarrow \infty} a_{\text {in }}<\lim \sup _{n \rightarrow \infty}\left(a_{\text {in }}+b_{\text {in }}\right)<1$ for $i=1, \ldots, m$. Then the sequence $\left\{x_{n}\right\}$ generated by (5) converges strongly to an element of $\mathscr{F}$.

Proof. Since $\lim _{n \rightarrow \infty}\left\|x_{n}-p\right\|$ exists for all $p \in \mathscr{F}$ by Lemma 6 , then, for any $p \in \mathscr{F}$ such that

$$
d\left(x_{n}, \mathscr{F}\right)=\left\|x_{n}-p\right\|
$$

we have that $\lim _{n \rightarrow \infty}\left\|x_{n}-p\right\|$ exists. It follows from (47) that $\lim _{n \rightarrow \infty} d\left(x_{n}, \mathscr{F}\right)$ exists. From condition (A)

$$
0 \leq f\left(d\left(x_{n}, \mathscr{F}\right)\right) \leq\left\|x_{n}-T_{i_{0}} x_{n}\right\|,
$$


where $\left\|x_{n}-T_{i_{0}} x_{n}\right\|$ is $\max _{1 \leq i \leq m}\left\|x_{n}-T_{i} x_{n}\right\|$. From Theorem $7 \lim _{n \rightarrow \infty}\left\|x_{n}-T_{i_{0}} x_{n}\right\|=0$. It then follows (48) that $\lim _{n \rightarrow \infty} f\left(d\left(x_{n}, \mathscr{F}\right)\right)=0$. By property of $f$, $\lim _{n \rightarrow \infty} d\left(x_{n}, \mathscr{F}\right)=0$. It also follows from (47) that $\lim _{n \rightarrow \infty}\left\|x_{n}-p\right\|=0$. Therefore $\lim _{n \rightarrow \infty} x_{n}=p \in \mathscr{F}$.

Now, we prove the weak convergence of iteration (5) for a family of asymptotically nonexpansive mappings in a uniformly convex Banach space.

Theorem 10. Let $X$ be a uniformly convex Banach space satisfying Opial's condition, and let $K$ be a nonempty closed convex subset of $X$. Let $T_{i}: K \rightarrow K(i=1,2, \ldots, m)$ be $m$ asymptotically nonexpansive mappings with sequence $\left\{k_{n}\right\}$, and let the sequences $\left\{a_{i n}\right\}_{n=1}^{\infty},\left\{b_{i n}\right\}_{n=1}^{\infty}$, and $\left\{u_{i n}\right\}_{n=1}^{\infty}, i=$ $1,2, \ldots, m$ be the same as in Theorem 7 . Then the sequence $\left\{x_{n}\right\}$ defined by (5) converges weakly to a common fixed point of $\left\{T_{i}: i=1, \ldots, m\right\}$.

Proof. It follows from Lemma 6 that $\lim _{n \rightarrow \infty}\left\|x_{n}-p\right\|$ exists. Therefore, $\left\{x_{n}-p\right\}$ is a bounded sequence in $X$. Then by the reflexivity of $X$ and the boundedness of $\left\{x_{n}\right\}$, there exists a subsequence $\left\{x_{n_{j}}\right\}$ of $\left\{x_{n}\right\}$ such that $x_{n_{j}} \rightarrow p$ weakly. By Theorem 7, $\lim _{n \rightarrow \infty}\left\|x_{n}-T_{i} x_{n}\right\|=0$, and $I-T_{i}$ is demiclosed at 0 for $i=1,2, \ldots, m$. So we obtain $T_{i} p=p$ for $i=$ $1,2, \ldots, m$. Finally, we prove that $\left\{x_{n}\right\}$ converges to $p$. Suppose $p, q \in w\left(\left\{x_{n}\right\}\right)$, where $w\left(\left\{x_{n}\right\}\right)$ denotes the weak limit set of $\left\{x_{n}\right\}$. Let $\left\{x_{n_{j}}\right\}$ and $\left\{x_{m_{j}}\right\}$ be two subsequences of $\left\{x_{n}\right\}$ which converge weakly to $p$ and $q$, respectively. Opial's condition ensures that $\omega\left(x_{n}\right)$ is a singleton set. It follows that $p=q$. Thus $\left\{x_{n}\right\}$ converges weakly to an element of $\mathscr{F}$. This completes the proof.

\section{Conflict of Interests}

The authors declare that there is no conflict of interests regarding the publication of this paper.

\section{Acknowledgment}

The authors are grateful for the very useful comments regarding detailed remarks which improved the presentation and the contents of the paper. The first author acknowledged that this paper was partially supported by Turkish Scientific and Research Council (Tübitak) Program 2224. This joint work was done when the first author visited University Putra Malaysia as a visiting scientist during 5th February-15th February, 2013. Thus, he is very grateful to the administration of INSPEM for providing him local hospitalities.

\section{References}

[1] K. Goebel and W. A. Kirk, "A fixed point theorem for asymptotically nonexpansive mappings," Proceedings of the American Mathmatical Society, vol. 35, pp. 171-174, 1972.

[2] S. C. Chang, Y. J. Cho, and H. Zhou, "Demi-closed principle and weak convergence problems for asymptotically nonexpansive mappings," Journal of the Korean Mathematical Society, vol. 38, no. 6, pp. 1245-1260, 2001.
[3] Y. J. Cho, H. Zhou, and G. Guo, "Weak and strong convergence theorems for three-step iterations with errors for asymptotically nonexpansive mappings," Computers and Mathematics with Applications, vol. 47, no. 4-5, pp. 707-717, 2004.

[4] M. A. Noor, "New approximation schemes for general variational inequalities," Journal of Mathematical Analysis and Applications, vol. 251, no. 1, pp. 217-229, 2000.

[5] M. O. Osilike and S. C. Aniagbosor, "Weak and strong convergence theorems for fixed points of asymptotically nonexpansive mappings," Mathematical and Computer Modelling, vol. 32, no. 10, pp. 1181-1191, 2000.

[6] S. Plubtieng, R. Wangkeeree, and R. Punpaeng, "On the convergence of modified Noor iterations with errors for asymptotically nonexpansive mappings," Journal of Mathematical Analysis and Applications, vol. 322, no. 2, pp. 1018-1029, 2006.

[7] J. Schu, "Weak and strong convergence to fixed points of asymptotically nonexpansive mappings," Bulletin of the Australian Mathematical Society, vol. 43, no. 1, pp. 153-159, 1991.

[8] B. Xu and M. A. Noor, "Fixed-point iterations for asymptotically nonexpansive mappings in Banach spaces," Journal of Mathematical Analysis and Applications, vol. 267, no. 2, pp. 444-453, 2002.

[9] C. E. Chidume and B. Ali, "Approximation of common fixed points for finite families of nonself asymptotically nonexpansive mappings in Banach spaces," Journal of Mathematical Analysis and Applications, vol. 326, no. 2, pp. 960-973, 2007.

[10] I. Yıldırım and M. Özdemir, "Approximating common fixed points of asymptotically quasi-nonexpansive mappings by a new iterative process," Arabian Journal for Science and Engineering, vol. 36, no. 3, pp. 393-403, 2011.

[11] J. Quan, S.-S. Chang, and X. J. Long, "Approximation common fixed point of asymptotically quasi-nonexpansive-type mappings by the finite steps iterative sequences," Fixed Point Theory and Applications, vol. 2006, Article ID 70830, 8 pages, 2006.

[12] J. W. Peng, "On the convergence of finite steps iterative sequences with errors asymptotically nonexpansive mappings," IAENG International Journal of Applied Mathematics, vol. 37, no. 2, 5 pages, 2007.

[13] H. Kizıltunç and S. Temir, "Convergence theorems by a new iteration process for a finite family of nonself asymptotically nonexpansive mappings with errors in Banach spaces," Computers and Mathematics with Applications, vol. 61, no. 9, pp. 24802489, 2011.

[14] K. K. Tan and H. K. Xu, "Approximating fixed points of nonexpansive mappings by the Ishikawa iteration process," Journal of Mathematical Analysis and Applications, vol. 178, no. 2, pp. 301-308, 1993.

[15] H.-K. Xu, "Inequalities in Banach spaces with applications," Nonlinear Analysis: Theory, Methods \& Applications, vol. 16, no. 12 , pp. 1127-1138, 1991. 


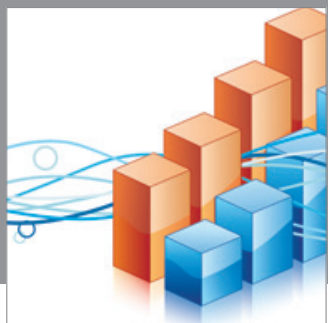

Advances in

Operations Research

mansans

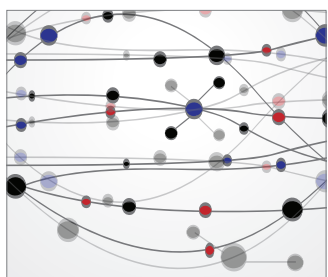

The Scientific World Journal
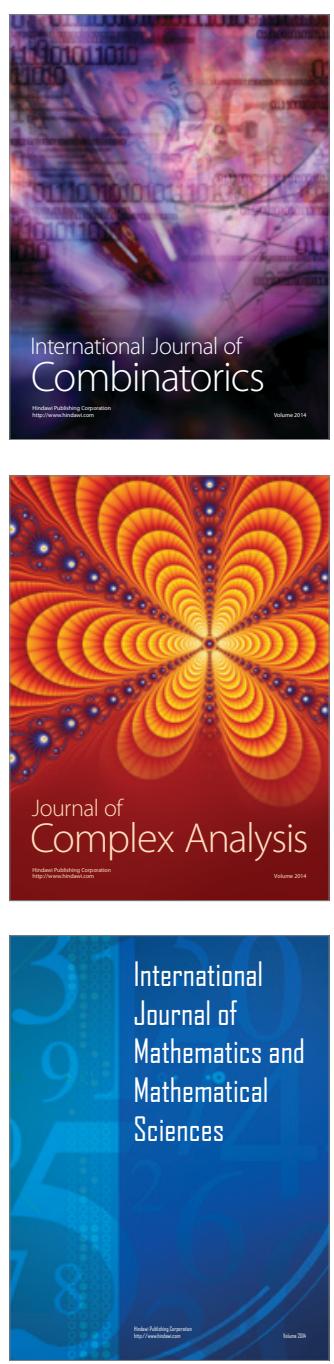
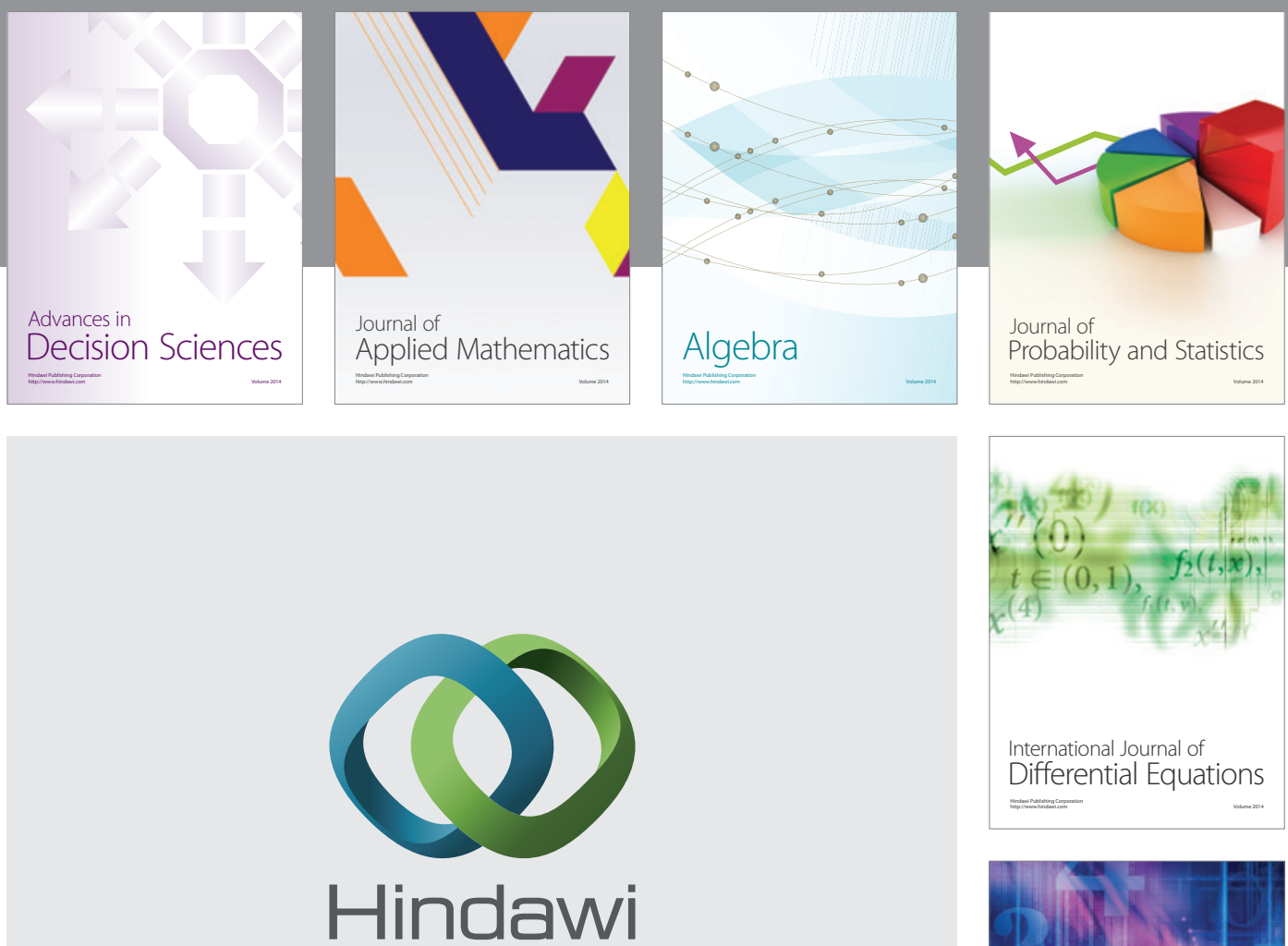

Submit your manuscripts at http://www.hindawi.com
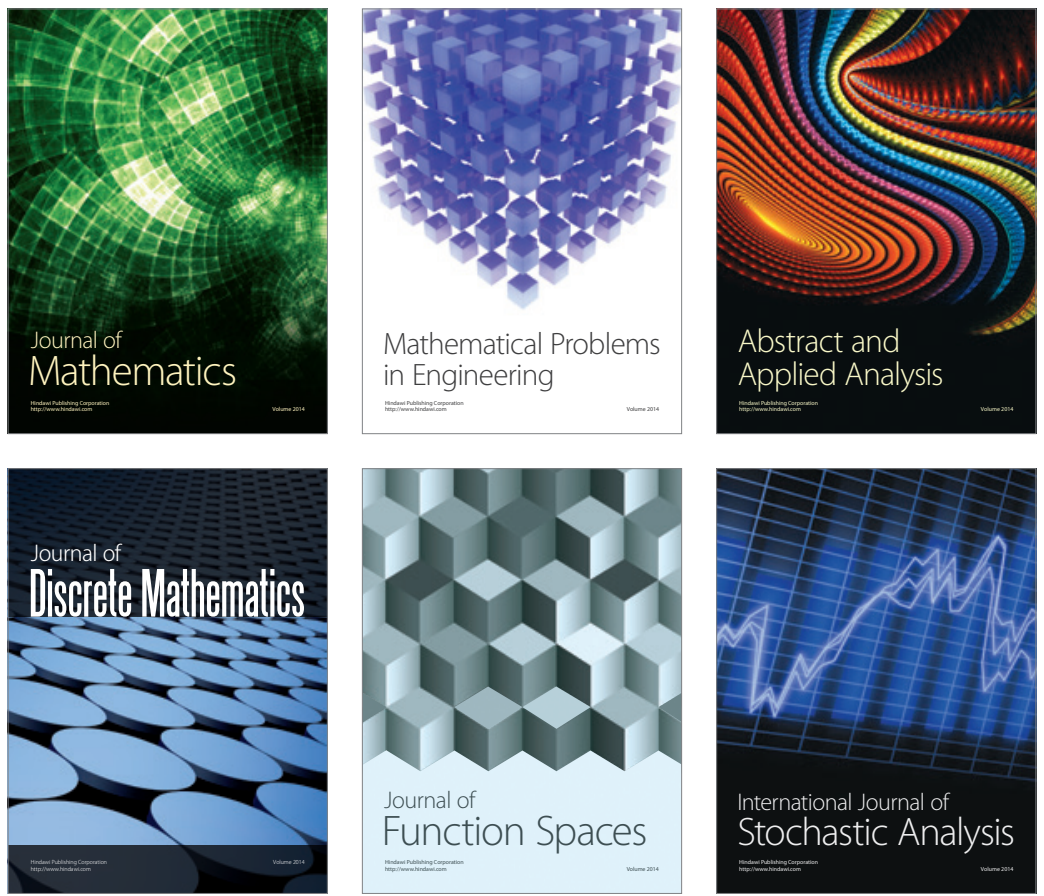

Journal of

Function Spaces

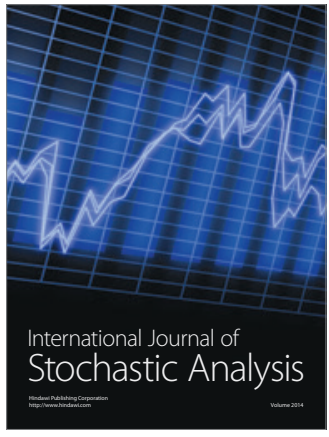

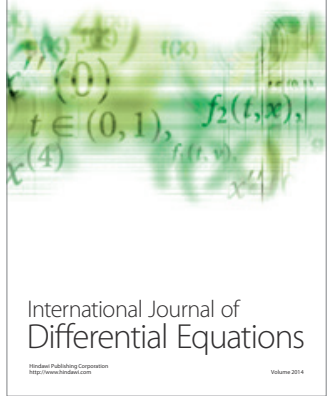
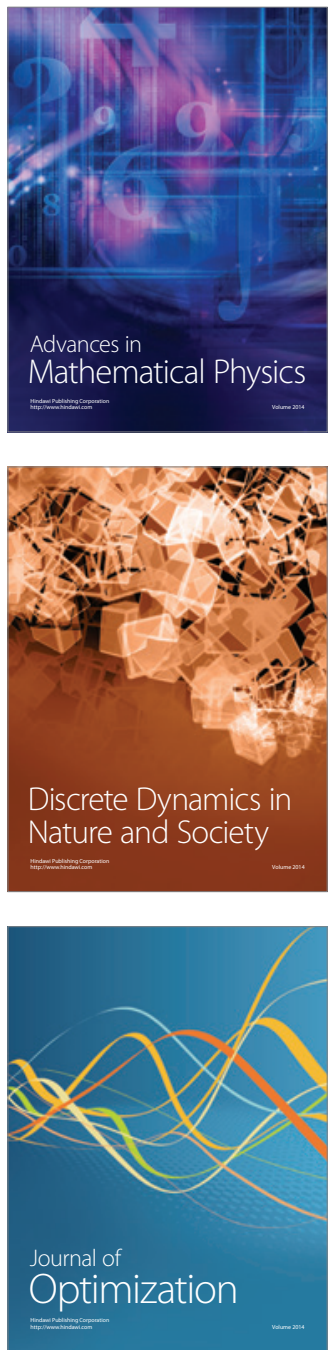